\title{
Selective Determination of Tryptophan-containing Peptides through Precolumn Derivatization and Liquid Chromatography Using Intramolecular Fluorescence Resonance Energy Transfer Detection
}

\author{
Makoto Yoshitake, Naoko SeJIMA, Hideyuki Yoshida, Kenichiro Todoroki, Hitoshi NohTa, ${ }^{\dagger}$ \\ and Masatoshi YAMAGUCHI
}

Faculty of Pharmaceutical Sciences, Fukuoka University, Nanakuma, Johnan, Fukuoka 814-0180, Japan

\begin{abstract}
A selective and sensitive fluorometric determination method for native fluorescent peptides has been developed. This method is based on intramolecular fluorescence resonance energy transfer (FRET) detection in a liquid chromatography (LC) system following precolumn derivatization of the amino groups of tryptophan (Trp)-containing peptides. In this detection process, we monitored the FRET from the native fluorescent Trp moieties (donor) to the derivatized fluorophore (acceptor). From a screening study involving 10 fluorescent reagents, we found that $o$-phthalaldehyde (OPA) generated FRET most effectively. The OPA derivatives of the native fluorescent peptides emitted OPA fluorescence $(445 \mathrm{~nm})$ through an intramolecular FRET process when they were excited at the excitation maximum wavelength of the Trpcontaining peptides $(280 \mathrm{~nm})$. The generation of FRET was confirmed through comparison with the analysis of a nonfluorescent peptide (C-reactive protein fragment (77-82)) performed using LC and a three-dimensional fluorescence detection system. We were able to separate the OPA derivatives of the Trp-containing peptides when performing LC on a reversed-phase column. The detection limits (signal-to-noise ratio $=3$ ) for the Trp-containing peptides, at a $20-\mu \mathrm{L}$ injection volume, were $41-180 \mathrm{fmol}$. The sensitivity of the intramolecular FRET-forming derivatization method is higher than that of the system that takes advantage of the conventional detection of OPA derivatives. Moreover, native non-fluorescent amines and peptides in the sample monitored at FRET detection are weaker than those of conventional fluorescence detection.
\end{abstract}

(Received April 11, 2007; Accepted May 16, 2007; Published August 10, 2007)

\section{Introduction}

Liquid chromatography (LC) coupled with a wide variety of detection methods such as ultraviolet (UV) detection, ${ }^{1}$ mass spectrometry (MS), ${ }^{2}$ electrochemistry ${ }^{3}$ and fluorometry ${ }^{4}$ have often been used for the determination of bioactive peptides. UV and electrochemical detections are convenient for the determination of peptides without derivatization. However, they are not sensitive and selective for peptides and, therefore, they require complicated clean-up procedures, such as liquid-liquid or solid-phase extractions. MS detection shows satisfactory structural specificity for peptides, but it needs expensive instrumentation and sophisticated operating skill. Fluorometric detection utilizing $o$-phthalaldehyde (OPA), ${ }^{4}$ dansyl chloride (DNS-Cl), ${ }^{5}$ fluorescamine (FA), ${ }^{6}$ and 4-fluoro7-nitro-2,1,3-benzoxadiazole ${ }^{7}$ as amine-reactive fluorogenic reagents have been reported. These methods, however, are not selective for peptides. On the other hand, highly selective LC methods based on specific fluorescent derivatization have also been reported. $N$-Terminal tyrosine and $N$-terminal tryptophan (Trp)-containing peptides have fluorescence derivatized with hydroxylamine-cobalt(II)-borate reagent $^{8}$ or glyoxal, ${ }^{9}$

$\doteqdot$ To whom correspondence should be addressed.

E-mail: nohta@fukuoka-u.ac.jp respectively. Although it is selective to the respective target peptides, it cannot be applied to the simultaneous determination of Trp-containing peptides, not only $\mathrm{N}$-terminals but also others.

In our previous research, we developed a highly selective determination method for indoleamines by LC with intramolecular fluorescence resonance energy transfer (FRET) detection. ${ }^{10}$ This method is based on the FRET-inducing derivatization of native fluorescent indoleamines and follows the detection of FRET from indoleamines (native fluorescent amines) to the derivatized fluorophore. FRET is the nonradiative transfer of excited-state energy from an initially excited donor fluorophore to an acceptor fluorophore. ${ }^{11}$ By using this characteristic, selective determination of indoleamines in human urine without special pretreatment has been achieved.

In the present study, we have developed a selective LC method for the determination of Trp-containing peptides by using intramolecular FRET-inducing derivatization (Fig. 1). One of the Trp-containing peptides, delta sleep-inducing peptide (DSIP, Trp-Ala-Gly-Gly-Asp-Ala-Ser-Gly-Glu), isolated from the venous blood of rabbits, ${ }^{12}$ has been used as a model compound. DSIP was discovered as a factor in slow-wave sleep. Several studies indicated that DSIP included various physiological and pathological reactions involving thermoregulation, locomotion, insomnia, pain, stress response, and circadian cycles. ${ }^{13-16}$ Thus, the determination of Trp- 


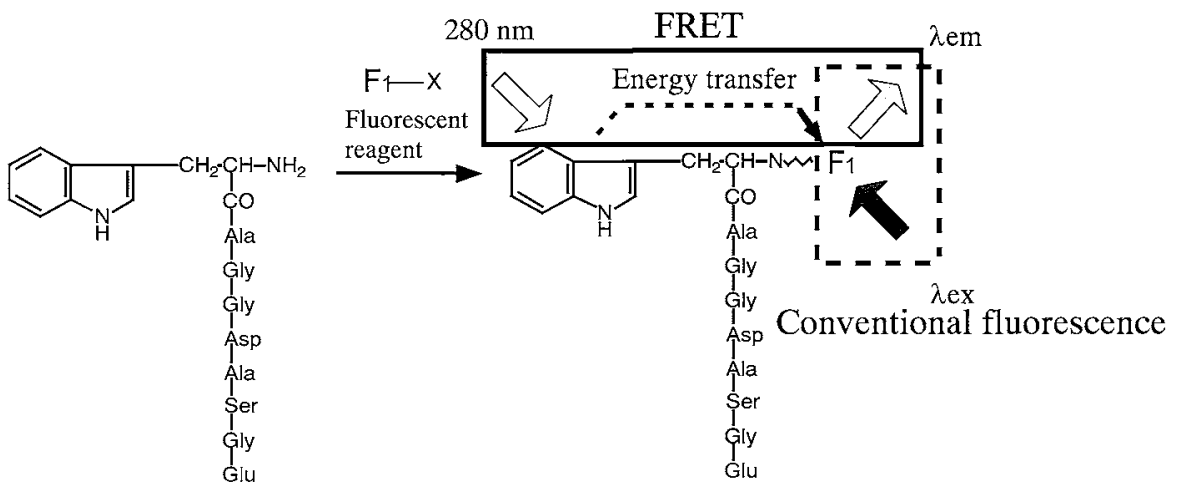

Fig. 1 FRET-inducing derivatization of DSIP.

containing peptides can provide a great deal of information to biochemical and clinical researchers. To find the most effective reagent, we screened 10 amine-reactive compounds for their induction of FRET in DSIP compared with that of the non-fluorescent peptide, C-reactive protein fragment (77 - 82) (C-RP, Val-Gly-Gly-Ser-Glu-Ile). In this paper, we validate the present method and compare it with previous methods.

\section{Experimental}

\section{Reagents and solutions}

DSIP was purchased from the Peptide Institute (Osaka, Japan). Osteostatin (Thr-Arg-Ser-Ala-Trp), C-RP, and Trp-Gly were obtained from Sigma (St. Louis, MO, USA). Gly-Gly-Trp and Gly-Gly-Cys were from Bachem (Bubendorf, Switzerland). DNS-Cl and OPA were purchased from Wako (Osaka, Japan). $N$-[4-(6-Dimethylamino-2-benzofuranyl)phenyl]isothiocyanate (DBPITC) and fluorescein-5-isothiocyanate (FITC) were obtained from Molecular Probes (Eugene, OR, USA). 4-Dimethylaminonaphthyl-1-isothiocyanate (DANITC), anthracene-2isothiocyanate (AITC), FA, 3-chlorocarbonyl-6,7-dimethoxy-1methyl-2(1H)quinoxalinone (DMEQ-COCl), and naphthalene2,3-dicarboxaldehyde (NDA) were from Acros (Geel, Belgium), Molecular BioSciences (Boulder, CO, USA), Fluka (Buchs, Switzerland), Dojin (Kumamoto, Japan), and Tokyo Kasei (Tokyo, Japan), respectively. These fluorescence derivatization reagents were used without further purification. 4,5Dimethoxyphthalaldehyde (DMPA) was synthesized according to Bhattacharjee's method. ${ }^{17}$ All organic solvents were of LCgrade. The other chemicals were of the highest purity available and were used as received. Distilled water, further purified using a Milli-QII system (Millipore, Milford, MA, USA), was used to prepare all aqueous solutions.

Stock solutions $(10 \mathrm{mM})$ of the respective peptides were prepared in aqueous methanol $(50 \%, \mathrm{v} / \mathrm{v})$ and were stored at $4^{\circ} \mathrm{C}$. These solutions were stable for at least 2 weeks and were diluted further with methanol to the required concentrations prior to use. The solutions of OPA (50 mM in methanol) and 2-mercaptoethanol (2-ME, 0.1 M in methanol) were prepared before use and were used up within a day. The borate buffer $(\mathrm{pH} 9.0,0.1 \mathrm{M})$ was prepared by mixing $0.1 \mathrm{M}$ boric acid and $0.025 \mathrm{M}$ sodium tetraborate and was stored at $4^{\circ} \mathrm{C}$.

\section{Fluorescence derivatization}

Screening tests. DSIP was used as a model compound of Trpcontaining peptides. C-RP was used for comparison with a non- fluorescent peptide. The 10 fluorescent reagents selective for amino groups that are listed in Table 1 were used in screening for the degree of FRET induced upon derivatization. The peptides were derivatized with the reagents according to the reported procedures. ${ }^{18-25}$

Optimized OPA reaction conditions. To an aliquot $(50 \mu \mathrm{L})$ of a standard solution of peptides placed in a $1.5-\mathrm{mL}$ Reacti-vial (Pierce, Rockford, IL, USA) were added $0.1 \mathrm{M}$ borate buffer $(\mathrm{pH} 9.0,25 \mu \mathrm{L}), 50 \mathrm{mM}$ OPA $(25 \mu \mathrm{L})$ and $0.1 \mathrm{M}$ 2-ME $(25 \mu \mathrm{L})$. The mixture was left to stand at room temperature for $10-15 \mathrm{~min}$. After the addition of the mobile phase $(375 \mu \mathrm{L})$, a portion $(20$ $\mu \mathrm{L}$ ) of the resulting mixture was injected into the LC system. As a blank, the standard solution was replaced with methanol $(50 \mu \mathrm{L})$ and subjected to the same procedure.

\section{$L C$ detection system and its conditions}

LC system. The LC system consisted of a Jasco (Tokyo, Japan) PU-980 chromatograph pump, a Rheodyne (Cotati, CA, USA) Model 7125 syringe-loading sample injector equipped with a 20- $\mu \mathrm{L}$ sample loop, a Jasco DG-980-50 on-line degasser, a reversed-phase column Shodex Asahipak ODP $(250 \times 4.6 \mathrm{~mm}$ i.d.; particle size, $5 \mu \mathrm{m}$; Showa Denko, Tokyo, Japan), and a Hitachi (Tokyo, Japan) L-7485 spectrofluorometer fitted with a $12-\mu \mathrm{L}$ flow-cell. The slit widths of the excitation and emission monochromators were set at $15 \mathrm{~nm}$. Chromatograms were recorded on a Hitachi D-2500 integrator, and all peaks were quantified by the valley-to-valley or valley-to-baseline method. $L C$ conditions for screening tests. The derivatized DSIP and C-RP were separated using acetonitrile-methanol-water as the mobile phase under isocratic elution. The flow rate of the mobile phase was set at $0.8 \mathrm{~mL} / \mathrm{min}$, and the column temperature was ambient $\left(23 \pm 3^{\circ} \mathrm{C}\right)$. The fluorescence detector was operated at an excitation wavelength of $280 \mathrm{~nm}$; the emission wavelength was set to the fluorescence emission maximum of the fluorescent reagent (Table 1). For comparative studies, the conventional fluorescence of each derivative was monitored at its fluorescence excitation and emission maxima (Table 1).

Conditions for the detection of OPA derivatives. The OPAderivatized peptides were separated using $15 \mathrm{mM}$ phosphate buffer ( $\mathrm{pH} 7.0)$-acetonitrile $(3: 1, \mathrm{v} / \mathrm{v})$ as the mobile phase. The flow rate of the mobile phase was set at $0.8 \mathrm{~mL} / \mathrm{min}$, and the column temperature was ambient. The fluorescence detector was operated at excitation and emission wavelengths of 280 and $445 \mathrm{~nm}$, respectively. For comparative studies, the conventional fluorescence of OPA derivatives was monitored at excitation and emission wavelengths of 335 and $445 \mathrm{~nm}$, respectively. 
Table 1 Screening of reagents: comparative study of FRET-induction between the fluorescent derivatives of DSIP and C-RP upon LC using FRET and conventional fluorescence detection methods

\begin{tabular}{|c|c|c|c|c|c|c|c|}
\hline \multirow{3}{*}{$\begin{array}{c}\text { Reagent } \\
\left(\lambda_{\mathrm{ex}} / \lambda_{\mathrm{em}}\right) / \mathrm{nm}\end{array}$} & \multicolumn{3}{|c|}{ DSIP (fluorescent) } & \multicolumn{3}{|c|}{ C-RP (non-fluorescent) } & \multirow{3}{*}{$\begin{array}{c}\begin{array}{c}\text { Selectivity } \\
\text { factor }\end{array} \\
\text { (A/B) } \\
\text { (C/D) }\end{array}$} \\
\hline & \multicolumn{2}{|c|}{ Peak area } & \multirow[b]{2}{*}{$\mathrm{A} / \mathrm{B}$} & \multicolumn{2}{|c|}{ Peak area } & \multirow[b]{2}{*}{$\mathrm{C} / \mathrm{D}$} & \\
\hline & $\begin{array}{l}\text { (A) FRET } \\
\text { detection }\end{array}$ & $\begin{array}{l}\text { (B) Conventional } \\
\text { fluorescence } \\
\text { detection }\end{array}$ & & $\begin{array}{l}\text { (C) FRET } \\
\text { detection }\end{array}$ & $\begin{array}{l}\text { (D) Conventional } \\
\text { fluorescence } \\
\text { detection }\end{array}$ & & \\
\hline OPA $(335 / 445)$ & 100 & 78 & 1.28 & 4 & 10 & 0.40 & 3.21 \\
\hline DMPA (330/447) & 350 & 150 & 2.33 & 612 & 560 & 1.09 & 2.14 \\
\hline DANITC (330/460) & 43 & 36 & 1.19 & 72 & 240 & 0.30 & 3.97 \\
\hline DNS-Cl (350/522) & 696 & 973 & 0.72 & 35 & 86 & 0.41 & 1.76 \\
\hline DBPITC (348/425) & 227 & 395 & 0.57 & 124 & 416 & 0.30 & 1.93 \\
\hline AITC $(358 / 453)$ & 1632 & 192 & 8.50 & 8516 & 954 & 8.93 & 0.95 \\
\hline FA $(381 / 470)$ & 521 & 342 & 1.52 & 1027 & 575 & 1.79 & 0.85 \\
\hline DMEQ-COCl (400/480) & 229 & 1784 & 0.13 & 1047 & 5122 & 0.20 & 0.63 \\
\hline NDA $(460 / 490)$ & 1828 & 298 & 6.13 & 9325 & 1110 & 8.40 & 0.73 \\
\hline FITC (495/520) & 1102 & 1669 & 0.66 & 1021 & 1567 & 0.65 & 1.01 \\
\hline
\end{tabular}

Conditions for native fluorescence detection. The Trpcontaining peptides were separated without derivatization using acetonitrile-methanol-water as the mobile phase under isocratic elution. The fluorescence detector was operated at excitation and emission wavelengths of 280 and $335 \mathrm{~nm}$, respectively.

\section{Three-dimensional fluorescence detection system}

An Agilent (Palo Alto, CA, USA) 1100 series LC system was used to confirm the generation of FRET. It consisted of a binary pump, an on-line degasser, an auto-sampler, a column oven, and a programmable 3D spectrofluorometer equipped with an $8-\mu \mathrm{L}$ flow-cell. Other conditions, except for the detection conditions, were the same as those described in the previous section.

On-line fluorescence spectra were obtained using a reaction mixture comprising DSIP and C-RP. The excitation spectra were monitored at $250-400 \mathrm{~nm}$ when the detector was operated with an emission wavelength of $445 \mathrm{~nm}$; the emission spectra were monitored at $400-550 \mathrm{~nm}$ with excitation at 280 (for FRET) or $335 \mathrm{~nm}$ (for conventional fluorescence of OPA derivatives). The slit widths of both the monochromators were set at $20 \mathrm{~nm}$.

\section{Results and Discussion}

\section{Screening tests for FRET-forming derivatization reagent}

It was necessary that we could confirm whether or not FRET occurs between Trp-containing peptides and the derivatized fluorophore. Therefore, we examined the 10 screened fluorescent reagents for Trp-containing peptides and selectivity factor. In these screening tests, we used DSIP as a model Trpcontaining peptide, and, for comparison, we used C-RP as a non-fluorescent peptide.

Table 1 provides the fluorescence intensities (peak area) of DSIP (A) and of C-RP (C) in FRET detection and in conventional fluorescence detection ((B) and (D), respectively). In this table, we defined two factors, $\mathrm{A} / \mathrm{B}$ and $(\mathrm{A} / \mathrm{B}) /(\mathrm{C} / \mathrm{D})$, as indices of the sensitivity and selectivity factors, respectively. The sensitivity factor is the ratio of peak areas in the FRET detection relative to that of the conventional detection system; increasing this value implies that FRET detection provides a more intense signal than does conventional detection. As a result, the sensitivity factors of OPA, DMPA, and DANITC
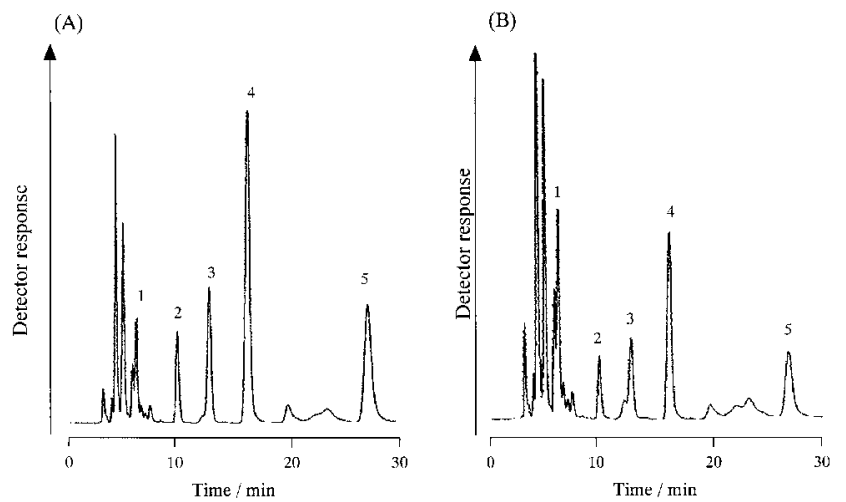

Fig. 2 Chromatograms obtained with peptides $(20$ pmol each on the column). (A) FRET and (B) conventional fluorescence detections monitored at excitation wavelengths of 280 and $335 \mathrm{~nm}$, respectively; emission wavelength, $445 \mathrm{~nm}$. Peaks: 1, Gly-Gly-Cys; 2, osteostatin; 3, DSIP; 4, Gly-Gly-Trp; 5, Trp-Gly; others, reagent blanks.

were $1.28,2.33$, and 1.19 , respectively. Among them, DMPA afforded the biggest sensitivity factor. However, it cannot be obtained from several commercial sources. The selectivity factor is the ratio of the sensitivity factor of DSIP with respect to that of C-RP; an increasing value suggests that DSIP provides a more intense signal than does C-RP in FRET detection. As a result, the selectivity factors of OPA, DMPA, and DANITC were $3.21,2.14$, and 3.97, respectively. Although DANITC afforded the biggest selectivity factor, many interfering peaks of DANITC were detected in the chromatogram when using it for simultaneous determination of Trp-containing peptides. On the other hand, the selectivity factors of AITC and NDA those sensitivity factors are greater than OPA and DANITC are less than 1.00. Thus, we selected OPA as the most suitable intramolecular FRET-inducing derivatization reagent for native fluorescent Trp-containing peptides.

\section{LC separation}

The best separations of the OPA derivatives of the Trpcontaining peptides (DSIP, osteostatin, Gly-Gly-Trp, Trp-Gly) and Gly-Gly-Cys were achieved within $30 \mathrm{~min}$ on a Shodex Asahipak ODP reversed-phase column using $15 \mathrm{mM}$ phosphate buffer ( $\mathrm{pH} 7.0)$-acetonitrile $(3: 1, \mathrm{v} / \mathrm{v})$ as the mobile phase under 


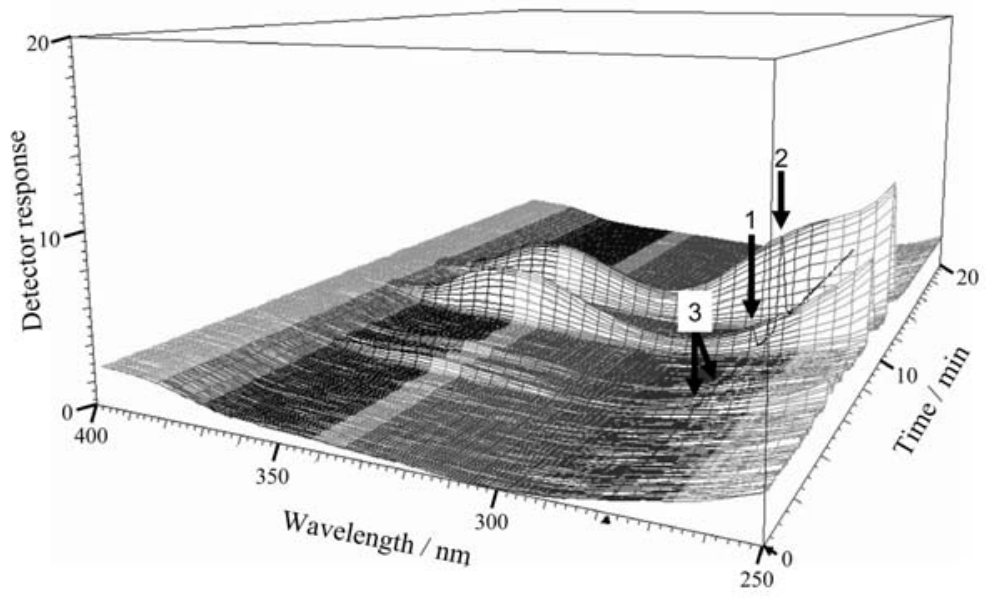

Fig. 3 Three-dimensional fluorescence excitation chromatogram obtained using the OPA-labeled DSIP and C-RP samples (400 pmol each on the column) at an emission wavelength at $445 \mathrm{~nm}$. Peaks: 1, C-RP; 2, DSIP; 3, unknown.

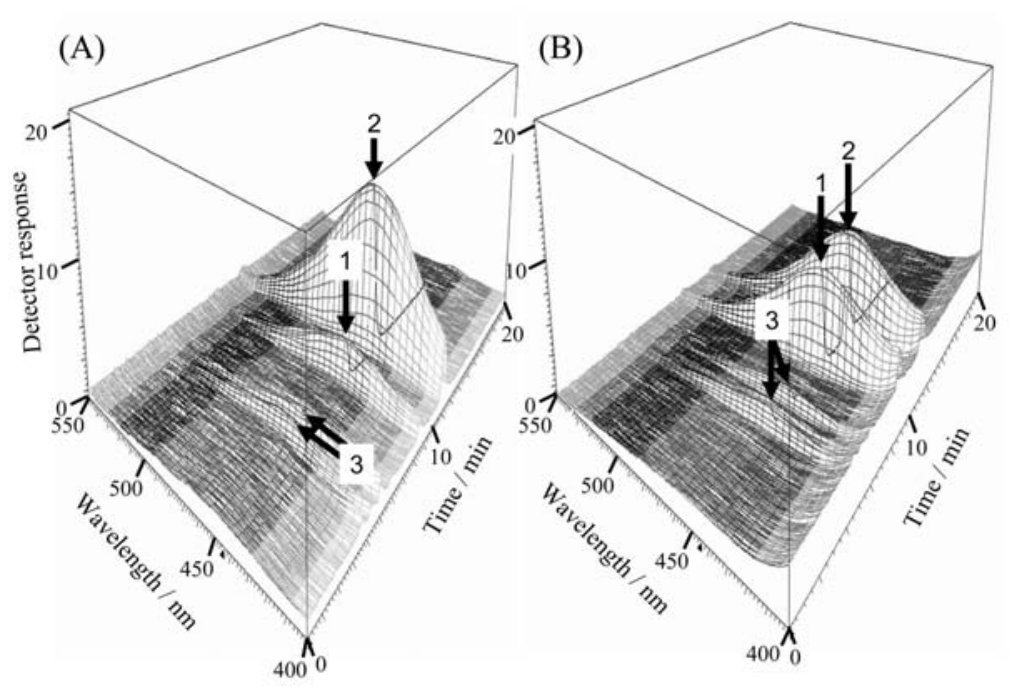

Fig. 4 Three-dimensional fluorescence emission chromatograms obtained using the OPA-labeled DSIP and C-RP samples at excitation wavelengths of (A) 280 and (B) $335 \mathrm{~nm}$. The peaks and amounts of the samples were the same as those listed in Fig. 3.

isocratic elution. Figure 2 illustrates the typical chromatograms that we obtained from the standard mixtures of Trp-containing peptides and Gly-Gly-Cys. The fluorescence intensities of Trpcontaining peptides that we monitored at excitation/emission wavelengths (nm) of 280/445 (FRET detection) were more intense than those monitored at 335/445 (conventional fluorescence detection). On the other hand, the fluorescence intensities of Gly-Gly-Cys and reagent blanks (the other native nonfluorescent amines in the sample and the working environment) monitored at FRET detection were weaker than those of conventional fluorescence detection. Thus, this intramolecular FRET-inducing derivatization method permits the selective and sensitive determination of Trp-containing peptides even if nonfluorescent amines are contained in the sample.

\section{Three-dimensional fluorescence chromatogram}

Figure 3 presents the three-dimensional fluorescence excitation chromatogram of DSIP and C-RP emitted at $445 \mathrm{~nm}$. The excitation maximum of DSIP was more intense than that of $\mathrm{C}-\mathrm{RP}$ at an excitation wavelength at $280 \mathrm{~nm}$ because FRET occurred between the native fluorescent moiety of DSIP and the labeled OPA.

Figure 4 illustrates the three-dimensional fluorescence emission chromatograms of DSIP and C-RP excited at $280 \mathrm{~nm}$ (A) and $335 \mathrm{~nm}(\mathrm{~B})$. The emission intensity of DSIP was more intense than that of C-RP at an excitation wavelength at $280 \mathrm{~nm}$, but they were equally intense at $335 \mathrm{~nm}$. The shape of the emission spectrum of DSIP in (A) was the same as that of (B). This can be understood by the following conditions: FRET between the native fluorescent moiety of DSIP and the labeled OPA should occur when DSIP is derivatized with OPA and the derivative is irradiated at the wavelength of the native fluorescent moiety. The blank peaks (peak 3 in Figs. 3 and 4), which are due to environmental amines, also became smaller upon FRET detection, as is the case with C-RP.

\section{Optimum conditions for OPA derivatization}

We performed optimization studies of the OPA derivatization process to maximize the FRET fluorescence peak areas for DSIP. Optimum derivatization conditions were examined 
Table 2 Detection limits ${ }^{\mathrm{a}}$ (fmol) of peptides with FRET, conventional, and native fluorescence detection methods

\begin{tabular}{|c|c|c|c|}
\hline \multirow{2}{*}{ Peptide } & \multicolumn{2}{|c|}{ OPA derivative } & \multirow{2}{*}{$\begin{array}{c}\text { Non-derivatized } \\
\text { peptide }\end{array}$} \\
\hline & FRET & $\begin{array}{l}\text { Conventional } \\
\text { fluorescence }\end{array}$ & \\
\hline DSIP & 100 & 220 & 680 \\
\hline Osteostatin & 180 & 280 & 220 \\
\hline Gly-Gly-Trp & 41 & 66 & 200 \\
\hline Trp-Gly & 110 & 200 & 110 \\
\hline
\end{tabular}

a. Defined as the amount of sample per injection volume $(20 \mu \mathrm{L})$ that gave a signal-to-noise ratio of 3 .

concerning OPA $(10-100 \mathrm{mM})$ and 2-ME (10-150 mM) concentrations, water-miscible organic solvents (dimethylsulfoxide, tetrahydrofuran, acetone, acetonitrile, methanol, ethanol, and $N, N$-dimethylformamide), reaction temperature $\left(0-80^{\circ} \mathrm{C}\right)$, and time $(1-90 \mathrm{~min})$; the conditions described in the Experimental section were selected. The OPA-derivatized Trp-containing peptides in the final reaction mixture were stable for at least $3 \mathrm{~h}$ in the dark at $4^{\circ} \mathrm{C}$.

\section{Linearity, precision, and detection limit}

The relationship between the amount of DSIP and the peak area was linear over the concentration range of $1-100 \mathrm{pmol}$ $(1.0,2.0,5.0,10,20,50$, and 100$)$ per $20-\mu \mathrm{L}$ injection volume; this range corresponds to $0.5-50 \mathrm{nmol} / \mathrm{mL}$ of the sample solution. The linear correlation coefficient $(n=3)$ was $>0.999$ for DSIP.

We established the within-day precision through repeated determinations of the DSIP standard $(5 \mathrm{nmol} / \mathrm{mL})$. The relative standard deviation $(n=5)$ of the peak area was $3.2 \%$.

Table 2 presents the detection limits that we obtained for the OPA derivatives detected using FRET wavelength and conventional wavelength and for the underivatized nativefluorescent wavelength of the Trp-containing peptides. The detection limits (per 20- $\mu \mathrm{L}$ injection volume; signal-to-noise ratio $=3$ ) of the native fluorescent peptides when using FRET derivatization were in the range of $41-180 \mathrm{fmol}$; this range is 2 times lower than that obtained when using conventional fluorescence detection. Thus, we achieved the sensitive determination of Trp-containing peptides upon their derivatization with OPA and subsequent FRET detection.

\section{Conclusion}

We have successfully applied a unique detection technique, one that takes advantage of intramolecular FRET-induced derivatization, to the selective determination of Trp-containing peptides. The derivatized Trp-containing peptides afforded FRET between their native fluorescent moieties and the OPAlabeled moieties. We observed that FRET detection provided more intense signals than did conventional fluorescence detection. Moreover, native non-fluorescent amines and peptides in the sample monitored at FRET detection were weaker than those of the conventional fluorescence detection. Therefore, we believe that the present method will be useful for biological and clinical investigations of Trp-containing peptides.

\section{Acknowledgements}

This study was supported in part by Grant-in-Aids for Scientific Research (B) (No. 17390013) and (C) (No. 12672100) from the Ministry of Education, Culture, Sports, Science, and Technology of Japan, and by fund (No. 046001) from the Central Research Institute of Fukuoka University. The authors thank Ms. N. Hiraki, Ms. T. Fusa, and Mr. M. Kubota for their excellent technical assistance.

\section{References}

1. J. River, R. McClintock, R. Galyean, and H. Anderson, $J$. Chromatogr., 1984, 303, 288.

2. P. Schulz-Knappe, M. Raida, R. He, B. Kleemeier, R. Richter, M. Schrader, L. Stadker, and W. Forssmann, Regul. Pept., 1996, 64, 171.

3. S. Mousa and D. Couri, J. Chromatogr., 1983, 267, 191.

4. H. Nakamura, C. L. Zimmerman, and J. J. Pisano, Anal. Biochem., 1979, 93, 423.

5. T. Chikuma, Y. Ogura, M. Kasamatsu, K. Taguchi, K. Mitsui, T. Kato, and M. B. Aguilar, J. Chromatogr., B, 1999, 728, 59.

6. W. Voelter and K. Zech, J. Chromatogr., 1975, 112, 643.

7. M. Gozdowska and E. Kulczykowska, J. Chromatogr., A, 2004, 807, 229.

8. M. Nakano, M. Kai, M. Ohno, and Y. Ohkura, J. Chromatogr., 1987, 411, 305.

9. M. Kai, E. Kojima, and Y. Ohkura, J. Chromatogr., 1993, 653, 235.

10. M. Yoshitake, H. Nohta, H. Yoshida, T. Yoshitake, K. Todoroki, and M. Yamaguchi, Anal. Chem., 2006, 78, 920.

11. J. R. Lakowicz, "Principles of Fluorescence Spectrometry", 1999, Kluwer Academic, New York.

12. G. A. Schoenenberger and M. Monnirer, Proc. Natl. Acad. Sci. U. S. A., 1977, 74, 1282.

13. M. V. Graf and A. J. Kastin, Peptide Rev., 1986, 7, 1165.

14. F. Bes, W. Hofman, J. Schuur, and B. C. Van, Neuropsychobiology, 1992, 26, 193.

15. E. Seifritz, M. J. Müller, G. A. Schönenberger, L. Trachsel, U. Hemmeter, M. Hatzinger, A. Ernst, P. Moore, and E. H. Trachsler, Peptide, 1995, 16, 1475.

16. E. M. Khvatova, N. A. Rubanova, I. A. Prudchenko, and I. I. Mikhaleva, FEBS Lett., 1995, 368, 367.

17. D. Bhattacharjee and F. D. Popp, J. Pharm. Sci., 1980, 69, 120.

18. K. Imai, J. Chromatogr., 1975, 105, 135.

19. P. M. Froehlich and T. D. Cunningham, Anal. Chim. Acta, 1978, 97, 357.

20. K. Muramoto, H. Kamiya, and H. Kawauchi, Anal. Biochem., 1984, 141, 446.

21. H. Tsuchiya, M. Tatsumi, N. Takagi, T. Koike, H. Yamaguchi, and T. Hayashi, Anal. Biochem., 1986, 155, 28.

22. H. Miyano, T. Nakajima, and K. Imai, Biomed. Chromatogr., 1987, 2, 139.

23. Y. F. Cheng and N. J. Dovichi, Science, 1988, 242, 562.

24. T. Kawasaki, T. Higuchi, K. Imai, and O. S. Wong, Anal. Biochem., 1989, 180, 279.

25. H. Yoshida, Y. Ohno, K. Todoroki, H. Nohta, and M. Yamaguchi, Anal. Sci., 2003, 19, 317. 